\title{
Photonic-band-gap engineering for volume plasmon polaritons in multiscale multilayer hyperbolic metamaterials
}

Zhukovsky, Sergei; Orlov, Alexey A.; Babicheva, Viktoriia E.; Lavrinenko, Andrei; Sipe, J. E.

Published in:

Physical Review A

Link to article, DOI:

10.1103/PhysRevA.90.013801

Publication date:

2014

Document Version

Publisher's PDF, also known as Version of record

Link back to DTU Orbit

Citation (APA):

Zhukovsky, S., Orlov, A. A., Babicheva, V. E., Lavrinenko, A., \& Sipe, J. E. (2014). Photonic-band-gap engineering for volume plasmon polaritons in multiscale multilayer hyperbolic metamaterials. Physical Review $A$, 90(1). https://doi.org/10.1103/PhysRevA.90.013801

\section{General rights}

Copyright and moral rights for the publications made accessible in the public portal are retained by the authors and/or other copyright owners and it is a condition of accessing publications that users recognise and abide by the legal requirements associated with these rights.

- Users may download and print one copy of any publication from the public portal for the purpose of private study or research.

- You may not further distribute the material or use it for any profit-making activity or commercial gain

- You may freely distribute the URL identifying the publication in the public portal 


\title{
Photonic-band-gap engineering for volume plasmon polaritons in multiscale multilayer hyperbolic metamaterials
}

\author{
Sergei V. Zhukovsky, ${ }^{1,2, *}$ Alexey A. Orlov, ${ }^{2}$ Viktoriia E. Babicheva, ${ }^{1,2,3}$ Andrei V. Lavrinenko, ${ }^{1}$ and J. E. Sipe ${ }^{4}$ \\ ${ }^{1}$ DTU Fotonik, Department of Photonics Engineering, Technical University of Denmark, Ørsteds Plads 343, DK-2800 Kongens Lyngby, \\ Denmark \\ ${ }^{2}$ ITMO University, Kronverksky Prospekt 49, St. Petersburg 197101, Russia \\ ${ }^{3}$ Birck Nanotechnology Center, Purdue University, 1205 West State Street, West Lafayette, Indiana 47907-2057, USA \\ ${ }^{4}$ Department of Physics and Institute for Optical Sciences, University of Toronto, 60 St. George Street, Toronto, Ontario, Canada M5S $1 A 7$
}

(Received 17 April 2014; published 2 July 2014)

\begin{abstract}
We study theoretically the propagation of large-wave-vector waves (volume plasmon polaritons) in multilayer hyperbolic metamaterials with two levels of structuring. We show that when the parameters of a subwavelength metal-dielectric multilayer (substructure) are modulated (superstructured) on a larger, wavelength scale, the propagation of volume plasmon polaritons in the resulting multiscale hyperbolic metamaterials is subject to photonic-band-gap phenomena. A great degree of control over such plasmons can be exerted by varying the superstructure geometry. When this geometry is periodic, stop bands due to Bragg reflection form within the volume plasmonic band. When a cavity layer is introduced in an otherwise periodic superstructure, resonance peaks of the Fabry-Pérot nature are present within the stop bands. More complicated superstructure geometries are also considered. For example, fractal Cantor-like multiscale metamaterials are found to exhibit characteristic self-similar spectral signatures in the volume plasmonic band. Multiscale hyperbolic metamaterials are shown to be a promising platform for large-wave-vector bulk plasmonic waves, whether they are considered for use as a kind of information carrier or for far-field subwavelength imaging.
\end{abstract}

DOI: 10.1103/PhysRevA.90.013801 PACS number(s): 42.70.Qs, 78.67.Pt, 81.05.Xj, 73.20.Mf

\section{INTRODUCTION}

Metamaterials have attracted avid scientific interest over the past decade because optical materials with properties rare or absent in nature can be artificially engineered. Notable examples include media with negative refraction [1] or giant optical activity [2] and so-called indefinite media, which exhibit hyperbolic dispersion relations [3-6]. The latter are a special case of extreme anisotropy where components of the diagonalized permittivity tensor have opposite signs [e.g., $\varepsilon=\operatorname{diag}\left(\varepsilon_{x}, \varepsilon_{y}, \varepsilon_{z}\right)$ with $\varepsilon_{x}=\varepsilon_{y}<0, \varepsilon_{z}>0$ for uniaxial anisotropic media]. With the introduction of these opposite signs, the dispersion relation

$$
\omega^{2} / c^{2}=k_{x}^{2} / \varepsilon_{z}+k_{y}^{2} / \varepsilon_{z}+k_{z}^{2} / \varepsilon_{x, y}
$$

changes from one of a conventional elliptical form to one of an exotic hyperbolic form [see Figs. 1(a) and 1(b)].

In the idealization that such a dispersion relation holds for all wave vectors, the isofrequency surface in the dispersion relation becomes unbounded [Fig. 1(b)]. As a result, waves with very large wave vectors $\left(k^{2} \gg \varepsilon_{x, y, z} \omega^{2} / c^{2}\right)$, which would normally be evanescent in any isotropic medium, can become propagating. Information carried by these high- $k$ modes with anomalously small wavelength $2 \pi / k$ can be used for farfield subwavelength imaging, as in the recent proposal of a hyperlens [7]. In addition, a multitude of high- $k$ modes greatly increases the local photonic density of states in the indefinite medium, bringing about a variety of new physical effects including broadband spontaneous emission enhancement $[8,9]$, anomalous heat transfer beyond the Stefan-Boltzmann

\footnotetext{
*sezh@fotonik.dtu.dk
}

limit [10], and an optical tabletop simulation platform for space-time phenomena such as metric signature transitions $[11,12]$.

Practical realization of indefinite media has been achieved over the past few years in the form of hyperbolic metamaterials (HMMs), which are highly anisotropic, subwavelength metaldielectric composites. Two geometries of HMMs have been preferred so far due to their simplicity in both modeling and fabrication. They are (i) metallic nanorod arrays embedded in a dielectric host [13-15] and (ii) metal-dielectric multilayers [8,9] shown in Fig. 1(c). In both structures, the behavior expected for indefinite media was experimentally confirmed [8,14], opening up many areas of theoretical and experimental research (for recent reviews see [16,17] and references therein). The finite size of the structure elements (rods or layers) puts an upper limit on the wave vectors $k$ that still satisfy Eq. (1) $[8,18,19]$. Still, the existence of such waves in HMMs has been clearly demonstrated [20,21]. Since these waves underlie the operation of a hyperlens and are crucial to other exotic physical properties of HMMs, it is very important to understand the physical nature of these waves and investigate the possible means of controlling their excitation and propagation.

The metal-dielectric composition of HMMs naturally leads one to suspect that high- $k$ modes are plasmonic in nature. Even though the exact mechanism of their formation has been debated [22,23], it is generally accepted that high- $k$ propagating waves must originate from surface plasmon excitations at individual metal-dielectric boundaries [20]. Because of this, names such as multilayer plasmons [24], Bloch plasmon polaritons [20,25], or volume plasmon polaritons (VPPs) [21] have been used by various groups. In our recent work [26] it was shown that VPPs originate from coupling between 

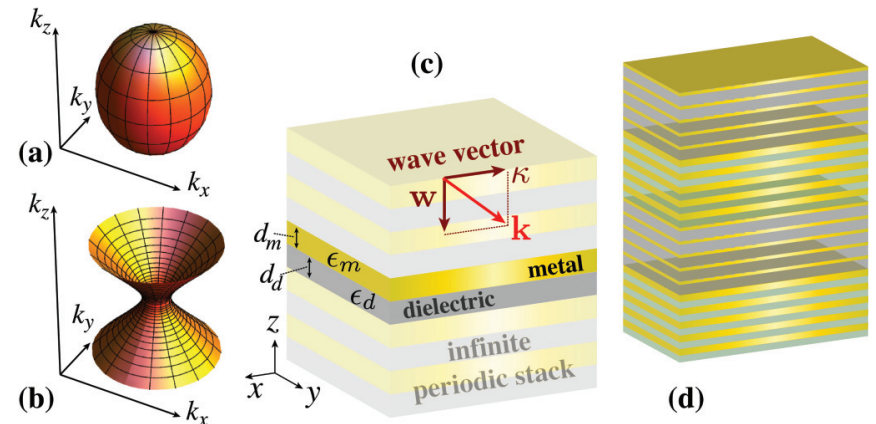

(d)

FIG. 1. (Color online) Dispersion relation of (a) a conventional anisotropic medium $\left(\varepsilon_{x, y, z}>0\right)$ with ellipsoidal isofrequency surface and (b) an indefinite medium $\left(\varepsilon_{x, y}<0\right.$ and $\left.\varepsilon_{z}>0\right)$ with hyperboloidal isofrequency surface. (c) Schematic of a periodic metaldielectric multilayer with the wave-vector decomposition $\mathbf{k}=w \hat{\mathbf{z}}+$ $\kappa \hat{\mathbf{x}}$ used in Sec. II (schematic in which both $w$ and $\mathbf{k}$ can be complex). (d) Schematic example of a multiscale metal-dielectric HMM, which is the main object of study in this paper.

short-range surface plasmon polaritons in the individual metal layers.

In order to utilize the full potential of VPPs as subwavelength information carriers for hyperlensing and other applications, it is necessary to understand how these waves can be guided and otherwise manipulated. A remarkable thing to observe is that they are bulk propagating waves, so they should be subject to the photonic-band-gap (PBG) effects similar to all other kinds of propagating waves. For an idealized model of homogeneous indefinite medium, a photonic structure can be imagined by imposing a modulation of medium parameters (e.g., $\varepsilon_{x, y}$ and $\varepsilon_{z}$ ), with the PBG properties depending on the geometry of that modulation. For example, a periodic stepwise modulation is expected to act as a photonic crystal for VPPs. In a realistic multilayer HMM, one can similarly envisage adding a wavelength-scale superstructure to an HMM, which already has a subwavelength substructure. In such multiscale HMMs, schematically shown in Fig. 1(d), VPP propagation is expected to be affected by the superstructure just as conventional light waves are controlled in photonic crystals. Just as unprecedented light control can be achieved in PBG structures, owing to a free choice of geometrical structures (e.g., periodic, coupled-cavity, quasiperiodic, fractal, etc.) and parameters, unprecedented control of plasmonic wave propagation should be possible by choosing an appropriate superstructure geometry in multiscale HMMs.

In this paper we demonstrate this multiscale approach by proposing proof-of-concept designs of Bragg reflectors and Fabry-Pérot resonators for high- $k$ bulk plasmons in multilayer metal-dielectric HMMs. Formation of PBGs in periodic multiscale multilayers is clearly seen in the numerically calculated Fresnel reflection coefficients in $k$ space. Breaking the periodicity in the superstructure is shown to result in cavity resonant modes. Making the structure totally nonperiodic increases the degree of freedom in using PBG effects to control the dispersion properties of HMMs. In particular, fractal multiscale HMMs are found to exhibit characteristic self-similar spectral features. Besides showing that high- $k$ waves can be directly controlled by PBG effects, the proposed approach is useful in designing HMM-based devices to engineer and probe the spontaneous emission rate of nearby atoms in the evanescent-wave domain.

The paper is organized as follows. In Sec. II we review the theoretical background on wave propagation in metaldielectric multilayer HMMs and discuss the dispersion relation of high- $k$ VPP waves in such multilayers. In Sec. III we introduce the concept of multiscale HMMs and show that VPPs can be manipulated by PBG effects. In particular, we demonstrate Bragg reflection and Fabry-Pérot resonances for VPPs in several periodic and nonperiodic multiscale geometries, including practically realizable designs. Section IV summarizes the paper.

\section{VOLUME PLASMON POLARITONS IN MULTILAYER HYPERBOLIC METAMATERIALS}

Consider a subwavelength periodic metal-dielectric multilayer as shown in Fig. 1(c), where the permittivities of dielectric and metal are $\varepsilon_{d}=\varepsilon_{d}^{\prime}+i \varepsilon_{d}^{\prime \prime}\left(\varepsilon_{d}^{\prime}>0\right)$ and $\varepsilon_{m}=$ $\varepsilon_{m}^{\prime}+i \varepsilon_{m}^{\prime \prime}\left(\varepsilon_{m}^{\prime}<0\right)$, respectively. The layer thicknesses are $d_{d}$ for the dielectric and $d_{m}$ for the metal and $\rho \equiv d_{m} /\left(d_{m}+d_{d}\right)$ denotes the metal filling fraction.

Any plane wave existing in such a multilayer can have its wave vector $\mathbf{k}$ represented as a sum of its in-plane component $\boldsymbol{\kappa}=k_{x} \hat{\mathbf{x}}+k_{y} \hat{\mathbf{y}}$ and its out-of-plane component $\mathbf{w}= \pm w \hat{\mathbf{z}}$. The former is constant across all layers due to the boundary conditions, so $\kappa=|\kappa|$ can be conveniently used as a labeling parameter for the waves. The out-of-plane component can take the value $\pm w$ in each layer (denoted by the subscript $m$ or $d$ ) with

$$
w_{m, d}=\sqrt{\left(\frac{\omega}{c}\right)^{2} \varepsilon_{m, d}-\kappa^{2}} .
$$

Generally we choose the square root of a complex number $\sqrt{z}$ such that $\operatorname{Im} \sqrt{z}>0$, taking $\operatorname{Re} \sqrt{z} \geqslant 0$ if $\operatorname{Im} \sqrt{z}=0$. Neglecting material absorption for now $\left(\varepsilon_{m, d}^{\prime \prime}=0\right)$, we can see that $w_{d}$ is real for $\kappa<\sqrt{\varepsilon_{d}} \omega / c$, corresponding to propagating waves within the light cone for the dielectric layers, or purely imaginary otherwise, corresponding to evanescent waves outside the light cone. For metal layers below the plasma frequency, $\varepsilon_{m}<0$, so $w_{m}$ is always imaginary.

If the layer thicknesses are subwavelength, the effectivemedium model is commonly used. In this case, the entire multilayer is regarded as a homogeneous medium with the permittivity tensor $\hat{\varepsilon}_{\text {eff }}=\operatorname{diag}\left(\varepsilon_{x}, \varepsilon_{y}, \varepsilon_{z}\right)$, where

$$
\varepsilon_{x}=\varepsilon_{y}=\rho \varepsilon_{m}+(1-\rho) \varepsilon_{d}, \quad \varepsilon_{z}^{-1}=\rho \varepsilon_{m}^{-1}+(1-\rho) \varepsilon_{d}^{-1} .
$$

In such an extremely anisotropic medium, the expression for $w$ of a $p$-polarized wave (for which surface plasmons can propagate along metal-dielectric interfaces) is [27]

$$
w_{\text {eff }}=\sqrt{\left(\frac{\omega}{c}\right)^{2} \varepsilon_{x}-\frac{\varepsilon_{x}}{\varepsilon_{z}} \kappa^{2}} .
$$

For layered HMMs one can choose the material parameters such that $\varepsilon_{x}<0$ and $\varepsilon_{z}>0$ (again, neglecting absorption for the moment). Then we see that the second term under the square root in Eq. (4) becomes negative and overrules the first 
(a)
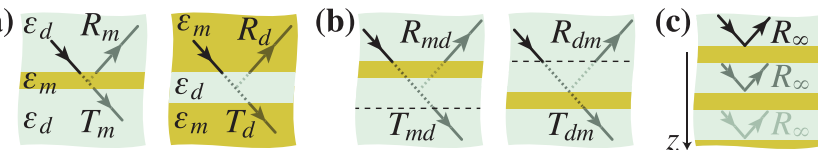

FIG. 2. (Color online) Illustration of the Fresnel reflection and transmission coefficient derivation for (a) a single metal or dielectric layers as in Eq. (7), (b) a metal-dielectric bilayer as in Eq. (10), and (c) a semi-infinite periodic metal-dielectric multilayer as in Eq. (11).

term for large enough $\kappa$. So the entire expression under the root becomes positive. Thus, the waves change from evanescent (imaginary $w$ ) to propagating (real $w$ ) at $\kappa=\kappa_{c}$ defined as $w_{\text {eff }}\left(\kappa_{c}\right)=0$.

Continuing to neglect absorption, we consider the expression of the Fresnel reflection coefficient for a boundary between a homogeneous dielectric and a medium described by the permittivity tensor in Eq. (3) [26],

$$
R_{\mathrm{eff}}=\frac{w_{d} \varepsilon_{x}-w_{\mathrm{eff}} \varepsilon_{d}}{w_{d} \varepsilon_{x}+w_{\mathrm{eff}} \varepsilon_{d}}
$$

In the region of large real $\kappa$, for which $w_{d}$ is imaginary, we see that real (rather than imaginary) $w_{\text {eff }}$ causes $R_{\text {eff }}$ to acquire a nonvanishing imaginary part [28]. In other words, $\operatorname{Im} R_{\mathrm{eff}}(\kappa)$ is nonzero for those values of $\kappa$ that correspond to propagating waves in the effective medium.

This correspondence is physically significant and can be extended to the case of actual multilayers. On the one hand, the dispersion relation of propagating Bloch waves in an infinite periodic metal-dielectric multilayer can be determined from the transfer-matrix method [29]. The transfer matrix of one period can be written as

$$
M_{1}=\frac{1}{T_{m}}\left[\begin{array}{cc}
T_{m}^{2}-R_{m}^{2} & R_{m} \\
-R_{m} & 1
\end{array}\right]\left[\begin{array}{cc}
e^{i w_{d} d_{d}} & 0 \\
0 & e^{-i w_{d} d_{d}}
\end{array}\right],
$$

where the reflection and transmission coefficients of a metal layer [see Fig. 2(a)] are given by the Airy formulas

$$
R_{m}=r_{d m}+\frac{t_{d m} r_{m d} t_{m d} e^{2 i w_{m} d_{m}}}{1-r_{m d}^{2} e^{2 i w_{m} d_{m}}}, \quad T_{m}=\frac{t_{d m} t_{m d} e^{i w_{m} d_{m}}}{1-r_{m d}^{2} e^{2 i w_{m} d_{m}}}
$$

the coefficients $r_{d m, m d}$ and $t_{d m, m d}$ are the interface coefficients for $p$-polarized waves determined by the Fresnel formulas

$$
\begin{array}{ll}
r_{m d}=\frac{w_{m} \varepsilon_{d}-w_{d} \varepsilon_{m}}{w_{m} \varepsilon_{d}+w_{d} \varepsilon_{m}}, & r_{d m}=\frac{w_{d} \varepsilon_{m}-w_{m} \varepsilon_{d}}{w_{d} \varepsilon_{m}+w_{m} \varepsilon_{d}} ; \\
t_{m d}=\frac{2 w_{m} \sqrt{\varepsilon_{m} \varepsilon_{d}}}{w_{m} \varepsilon_{d}+w_{d} \varepsilon_{m}}, & t_{d m}=\frac{2 w_{d} \sqrt{\varepsilon_{d} \varepsilon_{m}}}{w_{d} \varepsilon_{m}+w_{m} \varepsilon_{d}}
\end{array}
$$

and $w_{m, d}$ are given by Eq. (2). According to Bloch's theorem, the normal wave-vector component $k_{B}$ of the propagating Bloch wave with tangential wave-vector component $\kappa\left(k^{2}=\right.$ $\left.k_{B}^{2}+\kappa^{2}\right)$ is determined as $\cos \left[k_{B}\left(d_{m}+d_{d}\right)\right]=\left(\operatorname{Tr} M_{1}\right) / 2$, resulting in a well-known dispersion relation [22,23,30]

$$
\begin{aligned}
\cos \left[k_{B}\left(d_{m}+d_{d}\right)\right] \\
=\cos \left(w_{m} d_{m}\right) \cos \left(w_{d} d_{d}\right)-\frac{1}{2}\left(\frac{\varepsilon_{m} w_{d}}{\varepsilon_{d} w_{m}}+\frac{\varepsilon_{d} w_{m}}{\varepsilon_{m} w_{d}}\right) \\
\quad \times \sin \left(w_{m} d_{m}\right) \sin \left(w_{d} d_{d}\right) .
\end{aligned}
$$
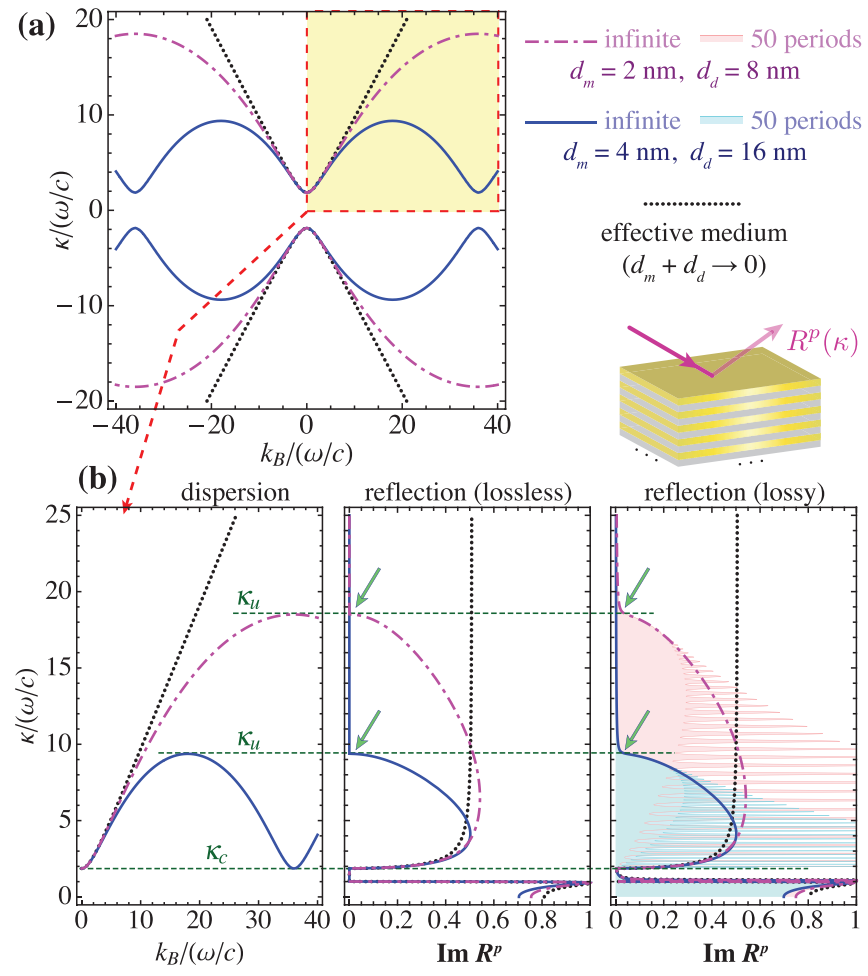

FIG. 3. (Color online) (a) Dispersion relation for high- $k$ Bloch waves in an infinite metal-dielectric HMM comprising alternating $\mathrm{Ag}$ and epoxy layers $\left(\varepsilon_{m}=-30.1+i \varepsilon_{m}^{\prime \prime}\right.$ and $\varepsilon_{d}=2.72$ for $\lambda=720 \mathrm{~nm}$ [30]) with $\rho=0.2$ and different layer thicknesses. (b) Enlarged view of a part of that dispersion relation along with the imaginary part of the Fresnel reflection coefficient $\operatorname{Im} R_{\infty}^{p}$ for a semiinfinite effective multilayer (see the inset) without losses $\left(\varepsilon_{m}^{\prime \prime}=0\right)$ and with losses $\left(\varepsilon_{m}^{\prime \prime}=0.41\right)$; the corresponding dependences of $\operatorname{Re} R_{\infty}^{p}(\kappa)$ are relatively featureless [28], slowly varying between 1 and 1.5 in the high- $\kappa$ range. The dotted lines denote the limiting case of the homogeneous effective anisotropic medium [Eq. (3)], corresponding to a multilayer with infinitely thin layers. The horizontal dashed lines denote the VPP band edges and faint green arrows highlight the differences in $\operatorname{Im} R_{\infty}^{p}$ for lossless vs real metal. The shaded areas in the rightmost plot correspond to a finite (50-period) multilayer as opposed to an infinite one.

The solution of this equation in the wave-vector space defines a band of propagating high- $k$ VPP waves [see Fig. 3(a)], which exists within certain limits $\kappa_{c}<\kappa<\kappa_{u}$. The lower band edge $\kappa_{c}$ is determined by the above-mentioned condition $w_{\mathrm{eff}}\left(\kappa_{c}\right)=$ 0 and coincides with the prediction of the effective-medium theory. Conversely, the upper band edge $\kappa_{u} \propto\left(d_{m}+d_{d}\right)^{-1}$ is associated with the breakdown of that approximation due to the finite layer thickness [28].

On the other hand, the reflection coefficient of a semiinfinite multilayer HMM can also be analytically determined. Applying the Airy formulas (7) one more time to a stack of two layers [metal and dielectric; see Fig. 2(b)] lets us express the transmission and reflection of such bilayers in terms of the single-layer reflection and transmission coefficients as [31]

$$
R_{m d}=R_{m}, \quad R_{d m}=R_{m} e^{2 i w_{d} d_{d}}, \quad T_{m d}=T_{d m}=T_{m} e^{i w_{d} d_{d}},
$$


with $R_{m}$ and $T_{m}$ given by Eq. (7). Since a semi-infinite structure remains unchanged when its outermost period is removed, its reflection coefficient $R_{\infty}$ must satisfy a quadratic equation

$$
R_{\infty}=R_{m d}+\frac{T_{m d} R_{\infty} T_{d m}}{1-R_{\infty} R_{d m}} .
$$

Solving this equation and choosing the solution for which the wave would be decaying, rather than growing, as $z$ increases [see Fig. 2(c)] we can determine $R_{\infty}$ [32].

In the absence of losses and for $\kappa>(\omega / c) \sqrt{\varepsilon_{d}}$, we can see that $w_{m, d}$ are purely imaginary and consequently $R_{m d, d m}$ and $T_{m d, d m}$ are purely real [see Eqs. (7) and (8)]. Then it can be shown that the existence condition for VPPs in the form $\left|\operatorname{Tr} M_{1}\right| \leqslant 2$ coincides with the condition $\mathscr{D} \leqslant 0$, where $\mathscr{D}$ is the discriminant of the quadratic equation (11), ensuring that its roots become complex even though its coefficients are real. This generalizes the relation between the nonzero imaginary part of the reflection coefficient at an interface and the existence of propagating waves beyond the interface in the evanescent-wave domain from the case of a single interface [as seen in Eqs. (4) and (5) above and as elaborated in the Appendix] to the case of a semi-infinite periodic multilayer HMM. Namely, we find that the imaginary part of $R_{\infty}$ is nonzero within the VPP band found by Bloch's theorem. Indeed, Fig. 3(b) shows that the range where $\operatorname{Im} R_{\infty} \neq 0$ is exactly $\kappa_{c}<\kappa<\kappa_{u}$, where propagating high- $k$ VPP waves were shown to exist. Elsewhere in the high- $\kappa$ range, $\operatorname{Im} R_{\infty}=$ 0 and no propagating solutions are allowed.

Even though rigorously derived for lossless, semi-infinite multilayers, this criterion is still a useful one if losses are present $\left(\varepsilon_{m, d}^{\prime \prime} \neq 0\right)$. Figure 3(b) shows that the abrupt appearance of nonzero $\operatorname{Im} R_{\infty}$ at the band edges is smeared [28] because the sharp distinction between evanescent and propagating waves can no longer be made if the materials are lossy. However, the general argument still persists that $\operatorname{Im} R_{\infty}$ is significantly nonzero when the Bloch solutions of the dispersion equation are propagating.

Moreover, including losses makes it possible to generalize the relation between nonzero $\operatorname{Im} R_{\infty}$ and the existence of propagating waves inside a finite multilayer structure. Note first that for lossless finite multilayer structures with $\kappa>$ $(\omega / c) \sqrt{\varepsilon_{d}}$ we see that all quantities entering the transfer matrix $M_{1}$ are real. Hence, the reflection coefficient of such a multilayer structure must be real too, with the exception of a discrete set of poles where the reflection coefficient diverges and its phase becomes indeterminate; these poles are known to signify the presence of guided modes inside the multilayer. As the number of layers in the structure increases, the number of poles grows accordingly, but it is only in the limit of an infinite multilayer that the transition from a discrete set to a continuous band can occur, as shown above. When it occurs, modes that are guided along the layers in a finite multilayer acquire a real $z$ component of the wave vector $\left[k_{B}\right.$ as given by Eq. (9)] and become propagating through the bulk of an infinite multilayer; it is for this reason that we refer to these waves as volume plasmon polaritons.

Thus, in the truly lossless case, finite multilayers only support surface waves with a discrete set of $\kappa>(\omega / c) \sqrt{\varepsilon_{d}}$, whereas infinite multilayers can additionally support bulk propagating waves (VPPs) in a continuous range of $\kappa$.

However, the presence of losses (even very minor ones) regularizes this opposition, transforming each discrete point into a narrow peak where $\operatorname{Im} R(\kappa) \neq 0$. When there are many layers in the structure, some of these peaks typically merge into a continuous band [see Fig. 3(b)], which is seen to approach the dependence $\operatorname{Im} R_{\infty}(\kappa)$ as the number of layers increases. Within this VPP band, there are waves inside the multilayer HMM that are quasipropagating in the sense that (i) their propagating character is primarily determined by the infinite-structure dispersion relation and is only weakly influenced by the number of layers in the structure, (ii) they undergo a much weaker attenuation than they would undergo in any homogeneous isotropic medium, and (iii) they become less attenuated if losses are lowered. In contrast, waves outside the VPP band remain strongly evanescent regardless of whether material losses are present. Hence we will be using the existence of $\operatorname{Im}[R(\kappa)]$ as a footprint, providing evidence for the existence of high- $k$ waves, or VPPs, in a range of HMM multilayer structures.

\section{MULTISCALE HYPERBOLIC METAMATERIALS}

Since VPPs are bulk Bloch plasmonic waves with propagating character, they have to be subject to the PBG effects just like any other kind of propagating waves. A straightforward idea is to apply these PBG effects to modulate the properties of a subwavelength multilayer HMM (in particular, the metal filling fraction $\rho$ ) on a larger length scale [Fig. 1(d)]. To distinguish the two scales, we will refer to the coarser, wavelength-scale modulation as the superstructure consisting of several superlayers; Fig. 4(a) displays two kinds of such superlayers (denoted A and B) with thicknesses $D_{A, B}$ and filling fraction $\rho_{A, B}$, respectively. The finer subwavelength periodic metal-dielectric structure within each superlayer, which gives rise to HMM properties, is called the substructure. Thus, each superlayer contains a certain number of subperiods $N_{A, B}=D_{A, B} /\left(d_{m}+d_{d}\right)$ or twice as many sublayers.

The resulting multiscale multilayer is expected to exert the same degree of control over VPP waves as the corresponding photonic multilayers control propagation of conventional electromagnetic waves. Hence, a periodic arrangement of

(a)
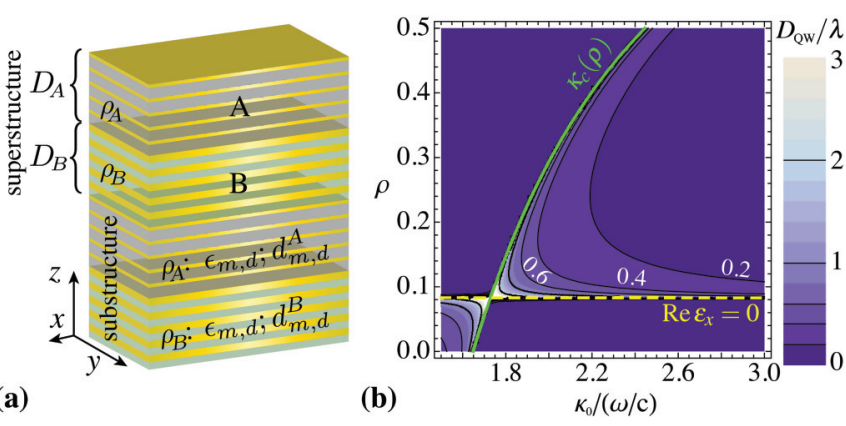

FIG. 4. (Color online) (a) Schematic of a multiscale HMM with periodic geometry, showing the division into the superstructure and substructure. (b) Dependence of the QW layer thickness $D_{\mathrm{QW}} / \lambda$ on filling fraction $\rho$ and the target PBG location $\kappa_{0}$ according to Eq. (12). The solid green line denotes the lower VPP band edge $\kappa_{c}(\rho)$ and the dashed yellow line marks the boundary of the HMM regime $\left(\operatorname{Re}\left[\varepsilon_{x}\right]=0\right)$. 
superlayers, where the layers denoted by A and B simply alternate, should result in a band gap for VPPs. In a further analogy with photonic multilayers, this band gap should forbid the propagation of waves with values of $\kappa$ around a certain midgap value $\kappa_{0}$, for which the superlayers are close to being a quarter wave (QW), i.e., their optical thickness should be close to one-quarter of the wavelength that corresponds to $w_{\text {eff }}\left(\kappa_{0}\right)$. In the remainder of this section, we will investigate the influence of the superstructure geometry on the VPP propagation in a variety of multiscale HMMs.

\section{A. Bragg reflectors and Fabry-Pérot resonators with thick-layer superstructure}

In order to demonstrate the multiscale concept, we first consider structures where the superstructure and substructure length scales are clearly separated, i.e., $\lambda / 4 \simeq D_{A, B} \gg d_{m, d}$ (or $N_{A, B} \gg 1$ ). Keeping in mind that the wavelength of high- $k$ VPPs, $\lambda \simeq 2 \pi / k$, can be anomalously small compared to the vacuum wavelength $\lambda_{0}=\omega / c$, it can be expected that the target $\kappa_{0}$ should be no more than several times larger than $\omega / c$. To overcome this apparent contradiction, we determine the thickness $D_{\mathrm{QW}}$ of a model homogeneous layer made of the effective medium [see Eq. (3)] and satisfying the QW condition for a given $\kappa_{0}$, i.e., a layer whose optical thickness with respect to a high- $k$ wave with certain parameters equals one-quarter of its effective wavelength. From Eq. (4),

$$
D_{\mathrm{QW}}=\frac{\lambda}{4}\left(\operatorname{Re}\left[\sqrt{\frac{\omega^{2}}{c^{2}} \varepsilon_{x}-\frac{\varepsilon_{x}}{\varepsilon_{z}} \kappa_{0}^{2}}\right]\right)^{-1} .
$$

We will use the term "QW layer" to denote a superlayer with thickness $D_{\mathrm{QW}}$ determined by Eq. (12).

The dependence of $D_{\mathrm{QW}}$ on $\rho$ and $\kappa_{0}$ is shown in Fig. 4(b). We are looking for the regime when we can achieve the band gap for waves with sufficiently large $\kappa_{0}$ using QW superlayers with sufficiently large thickness so that the superlayer can contain enough subperiods for the effective-medium theory to be applicable; in other words, we look for the values of $\rho$ for which $D_{\mathrm{QW}} / \lambda$ remains larger than $d_{m, d} / \lambda$ (and therefore have a clear separation of length scales $D_{\mathrm{QW}} \gg d_{m, d}$ ) for sufficiently large $\kappa_{0}>\kappa_{c}$. It can be seen in Fig. 4(b) that these conditions are satisfied for the filling fractions slightly above 0.08 , for which $\varepsilon_{x} \lesssim 0$.

Therefore, the design of a Bragg reflector for VPPs involves choosing two values of $\rho$ for the superlayers $\left(\rho_{A}\right.$ and $\left.\rho_{B}\right)$ and then using Eq. (12) to determine the superlayer thicknesses $D_{A, B}$. Following the example of Ni et al. [30] and choosing silver and epoxy as metal and dielectric materials, respectively $\left(\varepsilon_{m}=-30.1+0.41 i, \varepsilon_{d}=2.72\right.$ for $\left.\lambda=720 \mathrm{~nm}\right)$, we choose $\rho_{A}=0.1$ and $\rho_{B}=0.14$. To form a band gap at a target $\kappa_{0}=4 \omega / c$, we arrive at $D_{A}=116.4 \mathrm{~nm}$ and $D_{B}=65.8 \mathrm{~nm}$, approximately corresponding to $N_{A}=29$ and $N_{B}=16$ metaldielectric bilayers with $d_{m}+d_{d}=4 \mathrm{~nm}$ thickness [Fig. 5(a)].

Such layers would be very difficult to fabricate and are so thin that it is unlikely bulk optical constants could be used to characterize them. Nonetheless, as an initial analysis we theoretically characterize these nominal structures [Figs. 5(b)-5(g)] to help identify some of the essential physics. Considering first the semi-infinite superstructure $\left(M=10^{4}\right.$ periods in practical calculations) to suppress passband states and artificially lowering the imaginary part of the metal

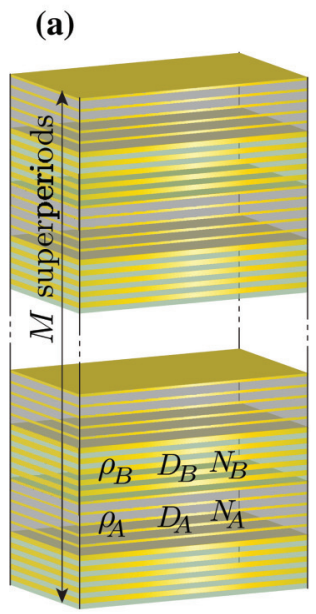

(b)

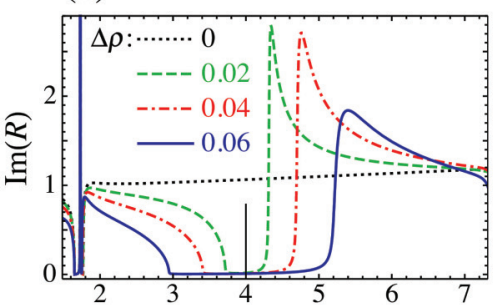

(c)

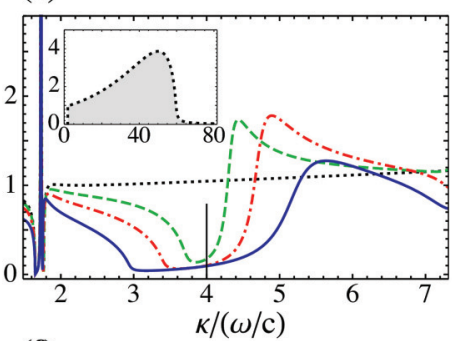

(e)

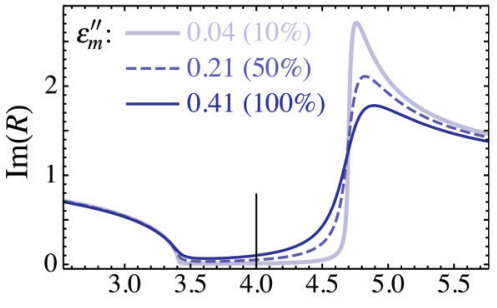

FIG. 5. (Color online) (a) Characterization of a multiscale Bragg reflector for high- $k$ waves shown schematically with the default geometry comprising a superstructure with $M=10^{4}$ periods of HMM layers with $\left(\rho_{A}+\rho_{B}\right) / 2=0.12$, the substructure period $d_{m}+d_{d}=4 \mathrm{~nm}$, and superlayer thickness $N_{A, B}$ determined from Eq. (12). The top row shows the function $\operatorname{Im}[R(\kappa)]$ for a varying depth of superstructure modulation $\left(\Delta \rho=\rho_{A}-\rho_{B}\right)$ for (b) $10 \%$ losses in silver $\left(\varepsilon_{m}^{\prime \prime}=0.04\right)$, (c) full losses in silver $\left(\varepsilon_{m}^{\prime \prime}=0.41\right)$, and (d) full losses in silver compensated by gain in the epoxy layers as described in [30] $\left(\varepsilon_{m}^{\prime \prime}=0.41\right.$ and $\left.\varepsilon_{d}^{\prime \prime}=-0.04\right)$; the dotted line shows the structure with no superstructure $\left(\rho_{A}=\rho_{B}=0.12\right)$, with the full high- $\kappa$ band shown in the inset of (c). The bottom row shows the dependence of $\operatorname{Im}[R(\kappa)]$ for the structure with $\rho_{A}=0.1, \rho_{B}=0.14$, $N_{A}=29$, and $N_{B}=16$ near the gap region for (e) varying amount of loss in silver $\varepsilon_{m}^{\prime \prime}$ for $\varepsilon_{d}^{\prime \prime}=0$, (f) varying amount of gain in dielectric $\varepsilon_{d}^{\prime \prime}$ for $\varepsilon_{m}^{\prime \prime}=0.41$, and (g) varying number of superperiods $M$. The inset in (f) compares the cases of $10 \%$ losses without gain and $100 \%$ losses with gain. The insets show $\operatorname{Im} R$ versus $\kappa /(\omega / c)$. (d)

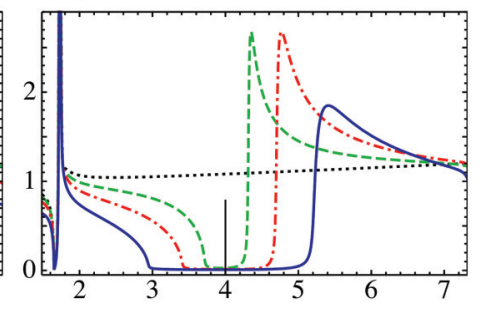

(g)

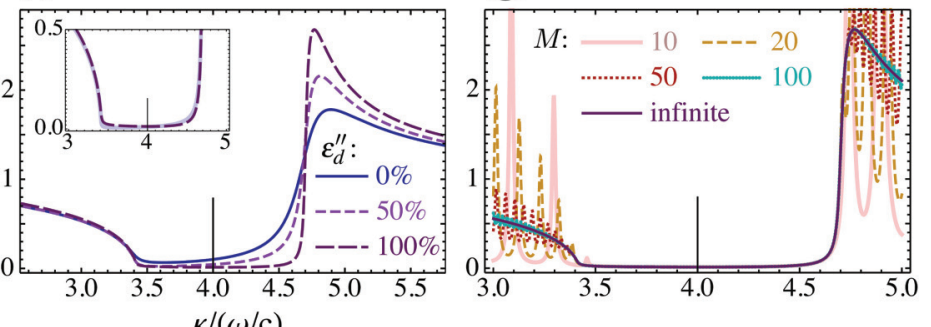


permittivity to $10 \%$ of its actual value, we clearly see a range of very low $\operatorname{Im} R$ around the target midgap $\kappa_{0}=4 \omega / c$ [Fig. 5(b)], signifying inhibited VPP propagation as described in Sec. II above. This range, or stop band, is seen to widen as the modulation depth of the filling fraction $\rho$ increases, which is characteristic of a PBG opening and confirms that VPPs indeed undergo Bragg reflection in a periodic-superstructure multiscale HMM.

Restoring the amount of losses in metal to their actual value [Fig. 5(c)], we see that the band gap is less pronounced but nevertheless quite visible. Compensating the loss in silver by incorporating optical gain in the dielectric layers of the HMM (setting $\varepsilon_{d}=2.72-0.04 i$, as was recently envisaged by $\mathrm{Ni}$ et al. [30]) restores the pronounced character of the band gap [see Fig. 5(d)]. The effects of loss and gain on the band-gap visibility are additionally highlighted in Figs. 5(e) and 5(f), where it is shown that adding gain to the dielectric layers indeed results in partial loss compensation. Since the working filling fractions are around 0.1 , so that $d_{d} \approx 10 d_{m}$, the negative imaginary component of $\varepsilon_{d}$ in the range of 0.04 is sufficient to compensate the loss in silver corresponding to $\varepsilon_{m}^{\prime \prime}=0.41$. Indeed, the inset in Fig. 5(f) demonstrates that the gap profile for the gain-compensated HMM is almost identical to that for the structure where loss is artificially reduced to $10 \%$. Finally, Fig. $5(\mathrm{~g})$ shows that reducing the number of superperiods does not change the location of the stop band for VPPs but strongly modifies the propagation of such waves in the surrounding passband of the high- $\kappa$ range, featuring multiple band-edgelike resonances.

Stacking a periodic Bragg reflector with its mirror image forms a structure with geometry $\mathrm{ABAB} \ldots \mathrm{ABB}$... ABAB [Fig. 6(a)], creating a half-wave defect or cavity (BB) in an otherwise periodic structure. By analogy with photonic multilayers, such a structure should function as a Fabry-Pérot resonator for VPPs, creating a narrow band of $\kappa \approx \kappa_{\text {res }}$ where the plasmon energy gets trapped in the cavity and the waves can tunnel through the structure despite the presence of a band gap. This feature should be observed as a sharp peak of $\operatorname{Im}\left[R\left(\kappa_{\mathrm{res}}\right)\right]$ inside the forbidden gap. Varying the thickness of the cavity layer (by adjusting the number of subperiods in it), it should be possible to tune the location of $\kappa_{\text {res }}$ across the band gap.

Such behavior is indeed observed in Fig. 6. The peak location is seen to shift as additional substructure periods are removed from or added to the defect layer, varying its thickness [Fig. 6(b)]. Note the stark contrast between the marked shift of the central resonance peak and the nearly absent shift of the remaining resonances in the wave-vector spectra. This means that the central peak is a Fabry-Pérot resonance, whereas the remaining peaks are not related to the cavity layer. For VPPs corresponding to the Fabry-Pérot resonance, it can be concluded that they are localized in the defect and guided within the $x-y$ plane.

Note that a much smaller number of periods in the superstructure is necessary for the appearance of the resonance peak than for the appearance of the band gap (see Fig. 5). For a larger number of periods in the superstructure it is seen that the guided high- $k$ VPP waves decouple from the incident wave, making them harder to characterize or interact with [Fig. 6(c)]. Also, similar to what we could see in Fig. 5, absorption in metal is highly detrimental: Without compensation, the resonance (a)
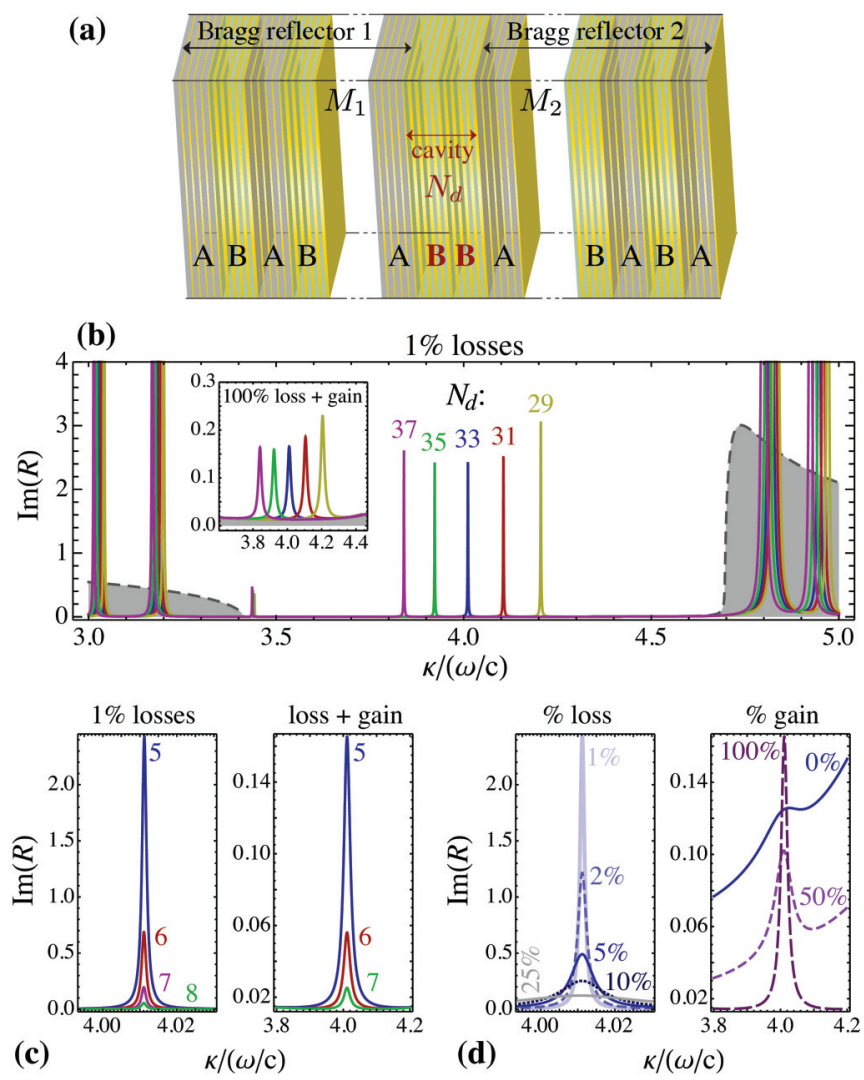

FIG. 6. (Color online) Characterization of a multiscale FabryPérot resonator for VPPs. (a) Schematic of the structure. (b) Dependence $\operatorname{Im}[R(\kappa)]$ for different thickness of the defect layer expressed in the number of superperiods $N_{d}$ for a superstructure with the same parameters as in Fig. 5 but with $M_{1}=M_{2}=5$ and $1 \%$ losses $\left(\varepsilon_{m}^{\prime \prime}=0.004\right)$; the inset shows the excerpt of the same dependence ( $\operatorname{Im} R$ versus $\kappa c / \omega$ ) for full losses in metal compensated by gain in the dielectric $\left(\varepsilon_{m}^{\prime \prime}=0.41\right.$ and $\left.\varepsilon_{d}^{\prime \prime}=-0.04\right)$. Also shown are the enlarged views of the cavity resonance peak under varying conditions in structures with (c) varying number of superperiods $M_{1}=M_{2}$ and (d) varying degree of loss and gain in the constituent materials.

peaks all but vanish when the imaginary part of $\varepsilon_{m}$ reaches $10 \%$ of its actual value. However, in the presence of gain the peaks are seen to reappear even with full metal losses [Fig. 6(d)]; the peaks are notably broadened, but their location is not affected [see the inset in Fig. 6(b)].

\section{B. Bragg reflectors and Fabry-Pérot resonators with thin-layer superstructure}

The approach of the previous section has a didactic advantage, with its easy separation of superstructure and substructure length scales; this makes the multiscale features that arise easy to understand. However, structures with $d_{m}+d_{d}=4 \mathrm{~nm}$, and indeed with $d_{m} \simeq 0.5 \mathrm{~nm}$, cannot be easily fabricated and, even if they could, the optical response of such thin layers would not likely be described by bulk dielectric constants. Further, even were fabrication possible it was shown that absorption is very detrimental to PBG effects, requiring either low-loss plasmonic materials [33] or loss compensation means [30] for 
(a)

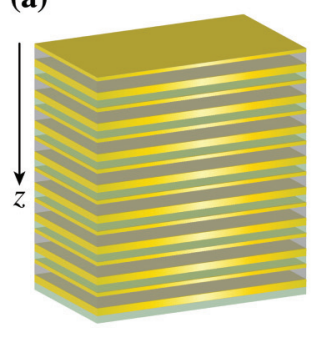

(b) small modulation depth

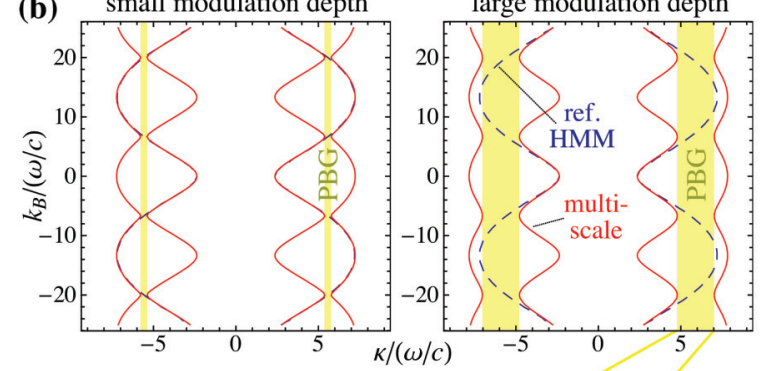

M: $-10 \quad$ - …… 20

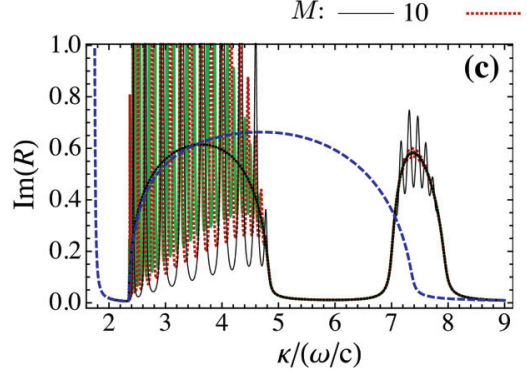

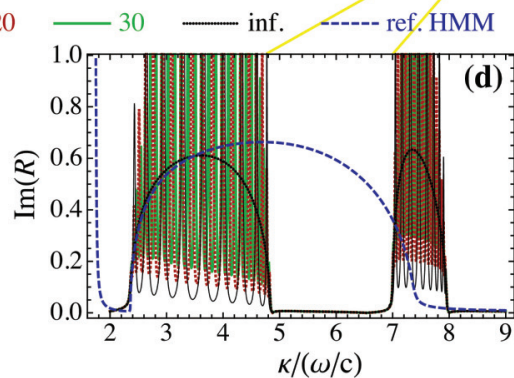

(e)

electric field distribution

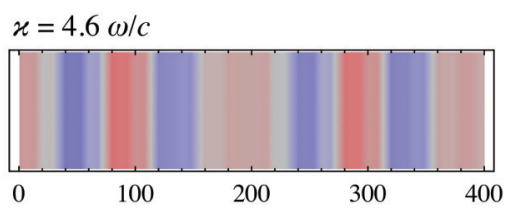

$x=6.0 \omega / c$

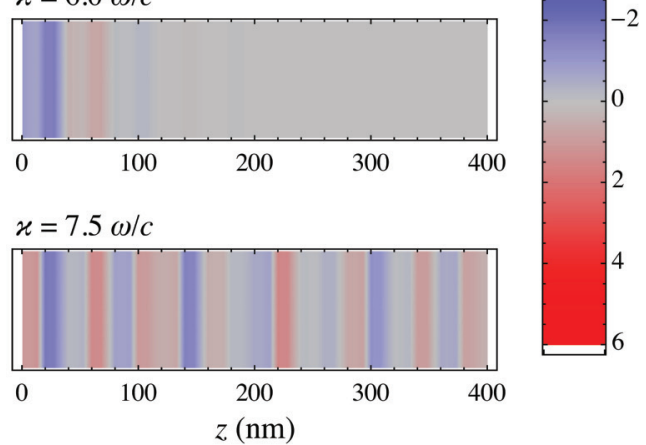

FIG. 7. (Color online) Characterization of a Bragg reflector for high- $k$ waves comprising a superstructure of 10-30 layers, each consisting of one subperiod $\left(N_{A}=N_{B}=1\right)$ with $\rho_{A}=0.60, \rho_{B}=0.34$, and $d_{m}+d_{d}=20 \mathrm{~nm}$. (a) Schematic of the structure. (b) Dispersion relation similar to Fig. 3 for an infinite double-periodic structure for small and large filling fraction modulation $\Delta \rho$. A plot of Im $R(\kappa)$ is shown for full metal losses (c) without and (d) with gain in the dielectric. The dashed line shows the reference VPP band from a HMM with average $\rho_{A}=\rho_{B}=0.473$. (e) Electric-field distribution in the structure with 20 periods for $\kappa c / \omega$ equal to 4.6 (below the band gap), 6.0 (inside the band gap), and 7.5 (above the band gap).

the effects to be observed. These disadvantages are worsened by the need for structures consisting of hundreds (if not thousands) of sublayers, leading even the most optimistic to despair of fabrication at any time in the near future. As a result, thick-superlayer structures for VPPs in multilayer HMMs can only be considered as proof-of-concept structures and are not viable from a practical point of view.

To alleviate these challenges in fabrication, we consider here the other extreme of the multiscale concept and analyze the structures where superlayers consist of just one subperiod $\left(N_{A}=N_{B}=1\right)$, allowing the metal and dielectric sublayers to be only moderately thin. In this regime, the subwavelength approximations will certainly fail [28], so Eq. (12) can no longer be regarded as an accurate prediction of a QW layer thickness. Instead, the structure can be considered as a periodic multilayer with a more complicated unit cell containing four layers [a double-periodic structure; see Fig. 7(a)]. Calculating its transfer matrix in a manner similar to Eq. (6) and applying Bloch's theorem along the lines of Sec. II, one can obtain the high- $k$ dispersion relation of such a double-periodic structure with an infinite number of periods. One can see [Fig. 7(b)] that if the filling fraction difference $\Delta \rho=\rho_{A}-\rho_{B}$ is nonzero, the VPP band splits into two, leaving a gap between them, which widens as $\Delta \rho$ increases. Since we are no longer restricted by the condition $D_{A, B} \gg d_{m . d}$, we can consider a structure with thicker sublayers (e.g., $d_{m}+d_{d}=20 \mathrm{~nm}$ ), which would be far more realistic for fabrication. We are also free to work with a greater contrast of $\rho$ and, as seen in Fig. 7(b), a prominently wide band gap can be achieved for an example structure with $\rho_{A}=0.60$ and $\rho_{B}=0.34$. The gap location is now in the higher- $\kappa$ part of the wave-vector space, around $\kappa \simeq 5 \ldots 7 \omega / c$, and it turns out that Eq. (12) is still able to give a meaningful estimate of the gap location, predicting the midgap $\kappa$ to be $6.75 \omega / c$.

Figures 7(c) and 7(d) show the characterization of such a multiscale HMM with a different number of superperiods. We can see that a PBG for VPPs does form at the predicted location with clear separation between passbands and stop bands with as few as several tens of superlayers. Together with practically achievable values for the sublayer thicknesses, this makes the whole structure much more promising for experimental realization than the thick-superlayer counterparts. Finally, it can be seen that the PBG is still clearly pronounced with the realistic account for the losses in silver [Fig. 7(c)]. To make sure that the feature seen in Fig. 7(c) is actually a PBG for VPPs seen in Fig. 7(b), we compare the electric-field distribution at three different $\kappa$, namely, below, inside, and above the band gap [Fig. 7(e)]. The field distribution shows the evanescent vs extended character of the waves inside the HMM for $\kappa$ inside vs outside of the band gap, respectively.

By adding gain to the dielectric layers, the gap becomes even more clearly defined, approaching abrupt band edges characteristic for the lossless case [see Fig. 3(b)]. This neartotal loss compensation makes this structure a practically realizable candidate for a Fabry-Pérot resonator for VPPs. As before, a basic half-wave defect is formed by repeating one of superlayers twice or more simply by stacking a three-superperiod structure $(\mathrm{ABABAB})$ with its mirror image (BABABA), as seen in Fig. 8(a). Rather than varying the number of sublayers in the cavity region (which is not possible since $N_{A}=N_{B}=1$ and $N_{d}=2$ ), the resonance location can be tuned by adjusting the thickness of one of the metal layers in the cavity region. Indeed, the characterization in Fig. 8(b) shows the possibility of moving the VPP resonance peak across 
(a)

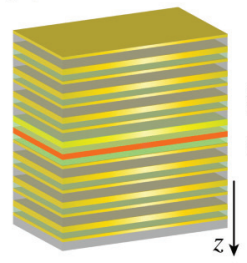

(b)

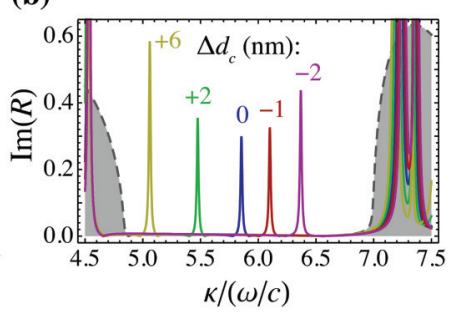

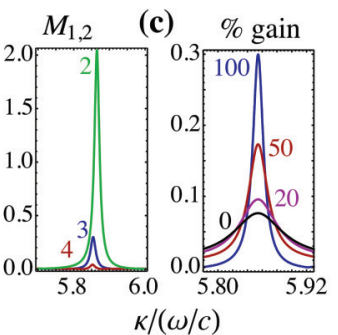

(d) $\Delta d_{c}=0$

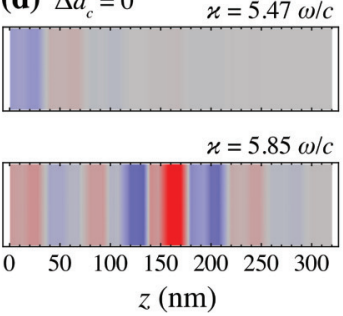

(e) $\Delta d_{c}=2 \mathrm{~nm}$
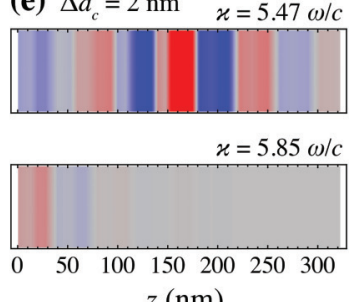

FIG. 8. (Color online) Characterization of a Fabry-Pérot resonator for high- $k$ waves comprising a superstructure with $M_{1}=M_{2}=3$ periods of two superlayers with the same substructure as in Fig. 7. (a) Schematic of the structure, highlighting the cavity region and variable-thickness metal layer in it. (b) Plot of $\operatorname{Im} R(\kappa)$ in the presence of gain for varying thickness of the central metal layer, $\Delta d_{c}$ being the thickness adjustment compared to the ideal half-wave defect. (c) Plot of $\operatorname{Im} R(\kappa)$ for $100 \%$ of metal losses and gain compensation in the dielectric. (c) Enlarged view of the peak for varying number of superperiods and degree of gain. The pictures at the bottom show the field distribution in the structures with (d) $\Delta d_{c}=0$ and (e) $\Delta d_{c}=2 \mathrm{~nm}$; in the top plots $\kappa=5.47 \omega / c$ (on peak for $\Delta d_{c}=2 \mathrm{~nm}$ ) and in the bottom plots $\kappa=5.85 \omega / c$ (on peak for $\left.\Delta d_{c}=0\right)$. The color scale is the same as in Fig. 7(e).

the band gap. The field distribution calculations show that when $\kappa$ matches the peak in the dependence $\operatorname{Im} R(\kappa)$, the structure features a Fabry-Pérot resonant mode localized near the cavity layer, whereas elsewhere in the band gap the waves in the HMM remain evanescent.

Figure 8(c) shows the dependence of the peak profile on the number of superperiods in the Bragg mirrors surrounding the cavity. As before, increasing this number beyond 4 makes the peak vanish by decoupling the localized VPP mode from the incident wave and reducing the amount of gain leads to broadening of the peak, again to the point of vanishing, due to the decrease of its $Q$ factor from around 300 in the presence of gain to around 50 without it. Even though these values are not spectacular from the resonator standpoint, we expect that the localization seen in Fig. 8(c) will contribute to the Purcell factor enhancement in the same way as does the judicious choice of the emitter position inside an HMM [19].

Overall, we see that thin-superlayer multiscale HMMs can exert the same PBG behavior on VPPs as their thicksuperlayer counterparts, but with a much smaller number of moderately thin layers, making such structures much more feasible for experimental realization. As a price to pay, the gap and/or resonance position can no longer be smoothly tuned by varying the number of subperiods in the superstructure; other methods such as departing from the QW condition in selected layers have to be used instead. We also see that for this thin-superlayer structure the gain compensation is quite successful in increasing the visibility of PBG effects. However, we refrain from far-reaching conclusions based on this result because the behavior of VPPs in the presence of gain strong enough to fully compensate (or even overcompensate) losses needs further investigation.

\section{Multigap and fractal Cantor multiscale HMMs}

As a final example, we briefly touch upon the possibility of band-gap engineering for VPPs by using more complicated superstructure geometries than simple periodicity. Note that doubling the number of subperiods in some superlayers of thin-superlayer structures (changing from $N_{A, B}=1$ to $N_{A, B}=2$ ) drastically influences the corresponding layers: They transform from quarter-wave-like to half-wave-like. This is expected to result in band-gap splitting. Indeed, Fig. 9 shows that by simple alteration of the superstructure periodicity, this multigap multiscale HMM can be realized. Aside from providing more versatility in the control over high- $k$ wave propagation, this effect allows us to push the band gap to the region with lower $\kappa$, which are easier to excite and are more prevalent in the emission of a realistic source (of finite size and/or located at a finite distance from the HMM [18,34]).

We can also envisage multiscale HMMs where superlayers lose their periodicity entirely while maintaining their longrange order, i.e., an ordered nonperiodic superstructure. Out of the many examples of such nonperiodic geometries [35], we will focus on a fractal Cantor-like structure [36-38]. These structures are known for scalable and self-similar features in their optical spectra closely related to their geometry [38-40].

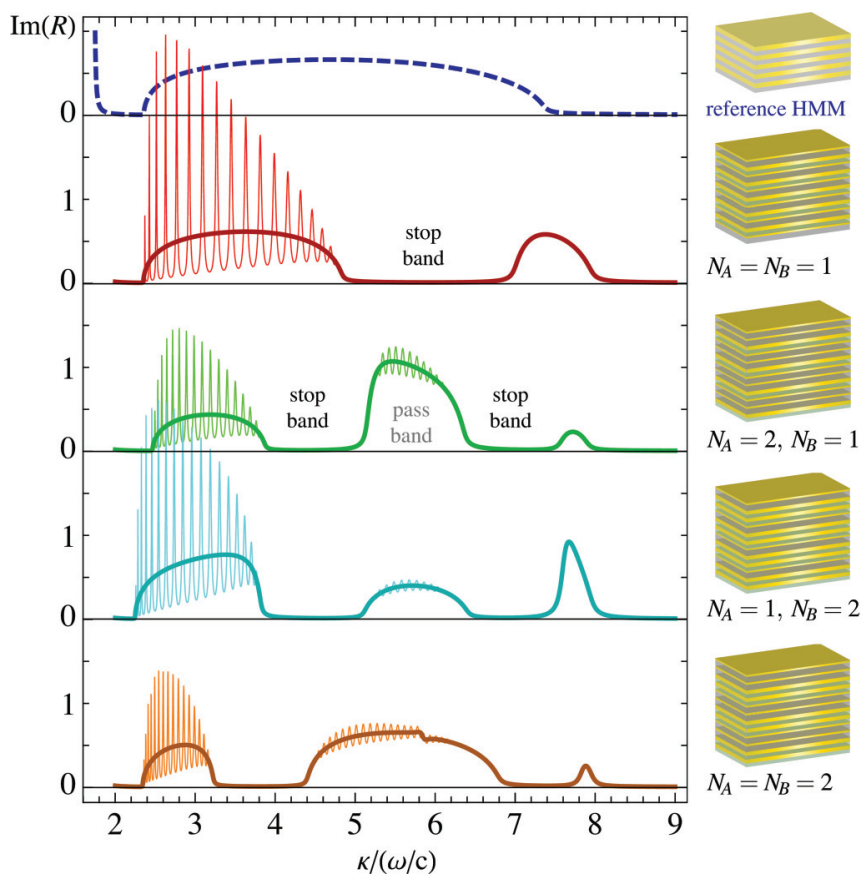

FIG. 9. (Color online) Schematics and high- $k$ band characterization of the structure with $N_{A}=N_{B}=1$ vs structures with doubled number of subperiods $\left(N_{A}=2, N_{B}=1 ; N_{A}=1, N_{B}=2 ; N_{A}=\right.$ $N_{B}=2$ ) with $M=20$ superperiods. Other parameters are the same as in Fig. 7. Full metal losses are considered. 

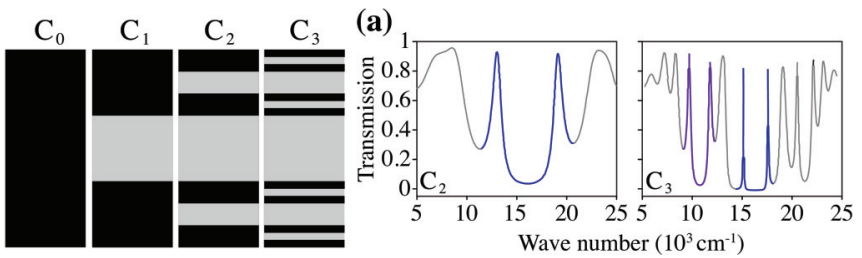

(b)

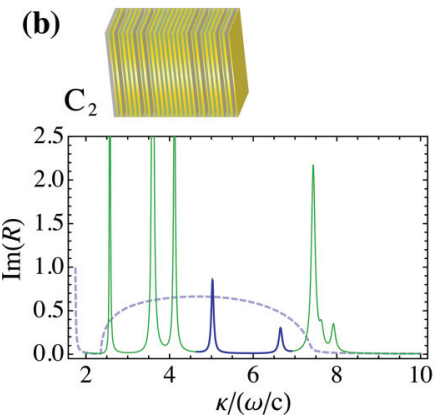

(c)
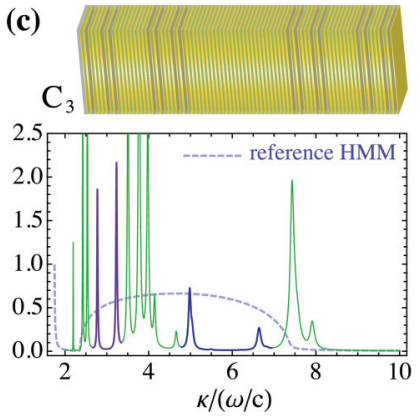

FIG. 10. (Color online) (a) Schematic of the middle-third Cantor section algorithm and an example of the optical spectrum of a Cantor multilayer [38,39]. (b) Schematic and high- $k$ band characterization of a second-generation Cantor structure $\left(C_{2}, 9\right.$ superlayers). (c) Same as (b) but for the third-generation Cantor structure $\left(C_{3}, 27\right.$ superlayers). The substructure geometry is the same as in Fig. 7. Full metal losses are considered.

Specifically, we will consider the simplest middle-third Cantor sequence [Fig. 10(a)], described by the inflation rules

$$
\mathrm{A} \rightarrow \mathrm{AAA}, \quad \mathrm{B} \rightarrow \mathrm{BAB},
$$

applied to a single layer of the type B (the seed) several times to form the Cantor structure of a given number of generation. This procedure yields the following sequence:

$$
\begin{aligned}
& C_{0}=\mathrm{B}, \quad C_{1}=\mathrm{BAB}, \quad C_{2}=\text { BABAAABAB }, \\
& C_{3}=\text { BABAAABABAAAAAAAAABABAAABAB } .
\end{aligned}
$$

It can also be written as a recurrent relation

$$
C_{n+1}=C_{n}(\mathrm{~A})^{3^{n}} C_{n},
$$

which underlies its geometrical self-similarity and gives rise to self-similar features in the optical spectra [Fig. 10(a)] [39].

Using the substructure geometry similar to the previous cases as the building blocks for A and B layers in Eqs. (13)-(15), we analyze the fractal multiscale HMMs corresponding to the second- and third-generation Cantor structures (containing 9 and 27 superlayers, respectively). The results are shown in Figs. 10(b) and 10(c). It can be seen that characteristic scalable signatures of the Cantor spectra can be observed for VPPs in the high- $\kappa$ region of the wave-vector space. These features are distorted compared to the traditional fractal multilayers because of the non-QW nature of the sublayers involved. Nonetheless, they are observed in a realistic structure with metal losses taken into account. So we can expect that known relations between geometrical and optical properties in deterministic nonperiodic multilayers should also be manifest in HMMs with corresponding superstructure geometry. As a result, we can make use of the extensive knowledge of
PBG properties of multilayers [37] to be able to control VPP propagation in multiscale HMMs with significant freedom.

\section{CONCLUSION}

To summarize, we have demonstrated that a multiscale approach can be used to control large-wave-vector, bulk plasmonic waves (volume plasmon polaritons) in multilayer metal-dielectric HMMs. As a proof of concept, we have proposed the design of Bragg reflectors and Fabry-Pérot resonators for these VPP waves. The designs consists of two levels of structuring: (i) a substructure of subwavelength metal and dielectric layers, responsible for creating hyperbolic dispersion, and (ii) a superstructure, which constitutes wavelength-scale variation of the metal filling fraction and exerts PBG effects on VPPs. Band gaps and resonances for VPPs have been demonstrated by examining the Fresnel reflection coefficient in the large-wave-vector region. More complicated superstructure geometries such as fractal Cantorlike multiscale HMMs have also been studied.

Along with proof-of-concept designs involving very large numbers of layers, more realistic thin-superstructure designs have been proposed, containing several tens of layers with thickness on the order of $10 \mathrm{~nm}$, which is within reach of modern fabrication technology. It has also been shown that mechanisms to mitigate material absorption, for example, by introducing gain-based compensation in dielectric layers [30], make PBG effects more pronounced in all of the considered structures.

Our results show that VPPs can be directly controlled by the PBG effects, which may be enabling for employing VPPs to transmit optical signals. Using a great variety of superstructure geometries in the proposed multiscale approach is promising in the design of HMM-based devices with a predetermined wave-vector-space distribution of bulk plasmonic waves. Such devices can be used in hyperlenses with tailored properties, as well as to probe and tailor light-matter interaction phenomena of nearby emitters (such as atoms and molecules) in the evanescent-wave domain.

\section{ACKNOWLEDGMENTS}

The authors wish to acknowledge inspiring discussions with K. Busch. This work received partial financial support from the People Programme (Marie Curie Actions) of the European Union's 7th Framework Programme FP7-PEOPLE2011-IIF under REA Grant Agreement No. 302009 (Project HyPHONE), as well as from the Natural Sciences and Engineering Research Council of Canada.

\section{APPENDIX: FRESNEL'S REFLECTION COEFFICIENTS IN THE EVANESCENT-WAVE DOMAIN}

In this Appendix we discuss the physical meaning of the Fresnel reflection coefficient at a plane interface between two media for such values of the in-plane component of the wave vector $\kappa$ that the waves in one or both of the media can be evanescent. The goal is to understand which attributes of the complex reflection coefficient (the real part, imaginary part, amplitude, and phase) have direct physical interpretation and to show that this interpretation changes significantly when the 
incident wave is evanescent rather than propagating. We need this knowledge in order to elucidate the relation between the existence of propagating waves in the HMM structures and the nonzero imaginary part of the reflection coefficients introduced in the text in Eqs. (5), (7), (8), (10), and (11), as well as through the transfer-matrix calculation procedure.

We use the interface between two nonmagnetic, isotropic media as an example and begin by recalling the expression for the reflection coefficient for a $p$-polarized wave at such an interface,

$$
r_{i j}(\kappa)=\frac{w_{i}(\kappa) \varepsilon_{j}-w_{j}(\kappa) \varepsilon_{i}}{w_{i}(\kappa) \varepsilon_{j}+w_{j}(\kappa) \varepsilon_{i}}
$$

assuming that there are no losses; therefore, we assume that $\varepsilon_{i, j}$ are purely real (positive or negative) and in turn that $w_{i, j}$, defined in the same way as in Eq. (2), are both one of the following:

$$
w_{i}(\kappa) \text { is } \begin{cases}\text { real and positive } & \text { for } \varepsilon_{i}>0, \kappa<\frac{\omega}{c} \sqrt{\varepsilon_{i}} \\ \text { positive imaginary } & \text { otherwise. }\end{cases}
$$

As mentioned in Sec. II, the first case corresponds to a wave that is propagating in medium $i$, whereas the second case corresponds to a wave that is evanescent in medium $i$. Depending on which of the cases of Eq. (A2) takes place for the two media ( $i$ and $j$ ) in Eq. (A1), four scenarios can be identified.

(i) Both $w_{i}$ and $w_{j}$ are real and hence $r_{i j}$ is real. This is the usual Snell refraction scenario: According to Eq. (A2), this is only possible if both $\varepsilon_{i}$ and $\varepsilon_{j}$ are non-negative so that the waves in both media are propagating, resulting in $\left|r_{i j}\right|<1$, which means that the transmitted wave carries some of the incident energy away.

(ii) $w_{i}$ is real but $w_{j}$ is imaginary; the incident and reflected waves are propagating but the transmitted wave is evanescent. This is the total reflection scenario: either conventional total internal reflection on a dielectric-dielectric interface beyond the critical angle $\left(\sqrt{\varepsilon_{j}}<\kappa c / \omega<\sqrt{\varepsilon_{i}}\right)$ or total reflection from a dielectric-metal interface $\left(\varepsilon_{j}<0<\varepsilon_{i}\right)$. Here the reflection coefficient is of the form $(a-i b) /(a+i b)$ and therefore $\left|r_{i j}\right|=1$, signifying that the transmitted evanescent wave transfers no energy away from the interface.

(iii) Both $w_{i}$ and $w_{j}$ are imaginary. Similar to (i) above, this means that $r_{i j}$ must be real; however, $\left|r_{i j}\right|$ can be below or above unity depending on the signs of $\varepsilon_{i, j}$. This is the scenario where all waves (incident, reflected, and transmitted) are evanescent and no energy transfer through the interface occurs. If we were to change the medium $i$ so as to support a propagating incident wave (e.g., by increasing its refractive index if it is a dielectric), this scenario would change to (ii).

(iv) Finally, $w_{i}$ is imaginary but $w_{j}$ is real. Similar to (ii) above, $r_{i j}$ is of the form $(a-i b) /(a+i b)$ and therefore has an imaginary part. This is the reversed total reflection scenario when the incident and reflected waves are evanescent but the transmitted wave is propagating; if we were to change the medium $i$ so as to support a propagating incident wave, this scenario would change to (i).

Suppose now that we know nothing about the nature of medium $j$ beyond the interface and only treat that region as a black box (or the sample). We still know, and can choose, the medium $i$ in front of the interface (the cladding) and we can perform some sort of ellipsometry-type reflectivity measurements on the interface. An important observation about the four scenarios listed above is that with the first two of them it is the amplitude of the reflection coefficient $\left|r_{i j}(\kappa)\right|$ that characterizes the sample's behavior at this particular $\kappa$; we can conclude that $\left|r_{i j}\right|=1$ means that there are no propagating waves in the sample (everything is totally reflected) and $\left|r_{i j}\right|<1$ means that there are propagating waves in the sample. On the other hand, the phase of the reflection coefficient (or individually its real or imaginary part) cannot be attributed such physical significance. Indeed, adding a cladding layer of thickness $d$ in front of the sample will not change anything in the physical system but will, according to Eq. (10) and Fig. 2(b), change $r_{i j}(\kappa)$ to $r_{i j}^{\prime}(\kappa)=r_{i j}(\kappa) e^{2 i w_{i}(\kappa) d}$, making its phase dependent on $d$ and therefore arbitrary.

On the other hand, if we apply the same line of reasoning to scenarios (iii) and (iv), we see that adding a similar cladding layer of thickness $d$ in front of the sample, with similar transformation $r_{i j}(\kappa) \rightarrow r_{i j}^{\prime}(\kappa)=r_{i j}(\kappa) e^{2 i w_{i}(\kappa) d}$, will change the amplitude rather than the phase of the reflection coefficient since we are in the regime where $w_{i}$ is imaginary; hence, it is $\left|r_{i j}\right|$ that can be made largely arbitrary. Therefore, it is now the phase of the reflection coefficient that characterizes the physics of the sample: $\arg r_{i j} \neq 0$ indicates that there are bulk propagating waves in the sample, while arg $r_{i j}=0\left(\right.$ real $\left.r_{i j}\right)$ unambiguously means that there are no such waves (although surface waves at the interface may still exist).

For practical purposes, whenever $r_{i j}$ is nonzero, we can introduce a modified criterion based on the imaginary part of $r_{i j}$ rather than on its phase. In these terms, $\operatorname{Im} r_{i j}=0$ (real $r_{i j}$ ) signifies the absence of bulk propagating waves in the sample, whereas $\operatorname{Im} r_{i j} \neq 0$ indicates their presence, as confirmed in Fig. 3. We stress here that the magnitude of $\operatorname{Im} r_{i j}$ still carries no direct physical significance in terms of characterizing the sample since $\left|r_{i j}\right|$ can be arbitrary; it is only whether it is zero or nonzero that is meaningful in the rigorous sense. However, we can relax our criterion somewhat, saying that $\operatorname{Im} r_{i j} \approx 0$ implies the absence of propagating waves in the sample and significantly nonzero $\operatorname{Im} r_{i j}$ implies their presence, as is demonstrated for the Bloch waves in Fig. 3(b). Vague as the words "significantly nonzero" are, the criterion in this form was demonstrated to be useful in a broad range of parameters, including complex multilayers and lossy structures, as confirmed by calculating the field distribution at corresponding $\kappa$; the only regime where we expect it to break down would be the case of high losses, where any nonarbitrary distinction between propagating and evanescent waves would be difficult.

We note finally that while the above analysis is carried out for an interface between two isotropic media, it remains valid if the sample is a homogenized HMM. Indeed, we see that Eq. (5) is essentially similar to Eq. (A1) as regards the applicability of Eq. (A2) and the subsequent reasoning; the sole reason for the explicit use of Eq. (A1) was to ease the explanation by being able to introduce a simple expression for $w_{j}$ using $\varepsilon_{j}$. Moreover, as outlined in Sec. II in the discussion of Eq. (11), the reasoning remains applicable to more complex samples such as infinite (and to some extent finite) multilayers. 
[1] J. Pendry, Opt. Express 11, 639 (2003).

[2] M. Kuwata-Gonokami, N. Saito, Y. Ino, M. Kauranen, K. Jefimovs, T. Vallius, J. Turunen, and Y. Svirko, Phys. Rev. Lett. 95, 227401 (2005).

[3] D. R. Smith, D. Schurig, J. J. Mock, P. Kolinko, and P. Rye, Appl. Phys. Lett. 84, 2244 (2004).

[4] D. R. Smith, D. Schurig, and P. Kolinko, J. Opt. Soc. Am. B 21, 1032 (2004).

[5] A. Degiron, D. R. Smith, J. J. Mock, B. J. Justice, and J. Gollub, Appl. Phys. A 87, 321 (2007).

[6] W. Yan, L. Shen, L. Ran, and J. A. Kong, J. Opt. Soc. Am. A 24, 530 (2007).

[7] Z. Jacob, L. V. Alekseyev, and E. Narimanov, Opt. Express 14, 8247 (2006).

[8] Z. Jacob, J.-Y. Kim, G. V. Naik, A. Boltasseva, E. E. Narimanov, and V. M. Shalaev, Appl. Phys. B 100, 215 (2010).

[9] Z. Jacob, I. I. Smolyaninov, and E. E. Narimanov, Appl. Phys. Lett. 100, 181105 (2012).

[10] C. Simovski, S. Maslovski, I. Nefedov, and S. Tretyakov, Opt. Express 21, 14988 (2013).

[11] I. I. Smolyaninov and Y.-J. Hung, J. Opt. Soc. Am. B 28, 1591 (2011).

[12] I. I. Smolyaninov and E. E. Narimanov, Phys. Rev. Lett. 105, 067402 (2010).

[13] M. A. Noginov, Yu. A. Barnakov, G. Zhu, T. Tumkur, H. Li, and E. E. Narimanov, Appl. Phys. Lett. 94, 151105 (2009).

[14] M. A. Noginov, H. Li, Yu. A. Barnakov, D. Dryden, G. Nataraj, G. Zhu, C. E. Bonner, M. Mayy, Z. Jacob, and E. E. Narimanov, Opt. Lett. 35, 1863 (2010).

[15] J. Kanungo and J. Schilling, Appl. Phys. Lett. 97, 021903 (2010).

[16] C. L. Cortes, W. Newman, S. Molesky, and Z. Jacob, J. Opt. 14, 063001 (2012).

[17] V. Drachev, V. A. Podolskiy, and A. V. Kildishev, Opt. Express 21, 15048 (2013).

[18] O. Kidwai, S. V. Zhukovsky, and J. E. Sipe, Opt. Lett. 36, 2530 (2011).
[19] I. Iorsh, A. Poddubny, A. Orlov, P. Belov, and Yu. Kivshar, Phys. Lett. A 376, 185 (2012).

[20] I. Avrutsky, I. Salakhutdinov, J. Elser, and V. Podolskiy, Phys. Rev. B 75, 241402(R) (2007).

[21] S. Ishii, A. V. Kildishev, E. Narimanov, V. M. Shalaev, and V. P. Drachev, Laser Photon. Rev. 7, 265 (2013).

[22] S. Feng, J. M. Elson, and P. L. Overfelt, Opt. Express 13, 4113 (2005)

[23] G. Rosenblatt and M. Orenstein, Opt. Express 19, 20372 (2011).

[24] J. Schilling, Phys. Rev. E 74, 046618 (2006).

[25] J. Elser, V. A. Podolskiy, I. Salakhutdinov, and I. Avrutsky, Appl. Phys. Lett. 90, 191109 (2007).

[26] S. V. Zhukovsky, O. Kidwai, and J. E. Sipe, Opt. Express 21, 14982 (2013).

[27] J. J. Saarinen and J. E. Sipe, J. Mod. Opt. 55, 13 (2008).

[28] O. Kidwai, S. V. Zhukovsky, and J. E. Sipe, Phys. Rev. A 85, 053842 (2012).

[29] A. Yariv and P. Yeh, Optical Waves in Crystals (Wiley, New York, 1983).

[30] X. Ni, S. Ishii, M. Thoreson, V. Shalaev, S. Han, S. Lee, and A. Kildishev, Opt. Express 19, 25242 (2011).

[31] S. V. Zhukovsky, Phys. Rev. A 81, 053808 (2010).

[32] S. V. Zhukovsky, L. G. Helt, D. Kang, P. Abolghasem, A. S. Helmy, and J. E. Sipe, Opt. Commun. 301-302, 127 (2013).

[33] P. R. West, S. Ishii, G. V. Naik, N. K. Emani, V. M. Shalaev, and A. Boltasseva, Laser Photon. Rev. 4, 795 (2010).

[34] A. N. Poddubny, P. A. Belov, and Yu. S. Kivshar, Phys. Rev. A 84, 023807 (2011).

[35] E. Maciá, Rep. Prog. Phys. 69, 397 (2006).

[36] X. Sun and D. Jaggard, J. Appl. Phys. 70, 2500 (1991).

[37] C. Sibilia, I. S. Nefedov, M. Scalora, and M. Bertolotti, J. Opt. Soc. Am. B 15, 1947 (1998).

[38] A.V. Lavrinenko, S.V. Zhukovsky, K.S. Sandomirski, and S.V. Gaponenko, Phys. Rev. E 65, 036621 (2002).

[39] S. V. Zhukovsky, A. V. Lavrinenko, and S. V. Gaponenko, Europhys. Lett. 66, 455 (2004).

[40] S. V. Zhukovsky and A. V. Lavrinenko, Photon. Nanostruct. 3, 129 (2005). 